\title{
South African Guidelines Excellence (SAGE): Clinical practice guidelines - quality and credibility
}

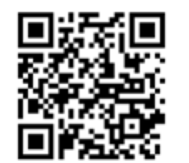

Over the past 15 years, the processes for developing clinical practice guidelines (CPGs) have shifted from their being written by experts (or based on expert opinion) to being largely written by methodologists. CPGs are quality improvement tools, and although they are presented in different ways, their aims are commonly to standardise care, improve its quality and safety, reduce wastage, decrease unnecessary costs, and improve access to care and patient outcomes. ${ }^{[1-3]}$

With the emergence of international collaborations such as the Guidelines International Network (G-I-N) ${ }^{[4]}$ there have been concerted attempts to standardise CPG writing practices across countries, to increase the credibility of the final products. ${ }^{[5-7]}$ Without adherence to rigorous guideline development and reporting standards, the considerable time and effort put into developing guidelines may be wasted, as intended users may not have confidence in the recommendations made.

South Africa (SA) is an emerging African leader in CPGs. However, there is room for improvement if SA CPG activities are to match global standards. ${ }^{[7]}$ In April 2014, the SAMJ signalled on its website the appointment of an editorial subcommittee whose specific mandate would be to review guidelines submitted for publication. The $S A M J$ has regularly published guidelines and recommendations for the management of a variety of conditions. These will in future be adjudicated using the AGREE II instrument (www.agreetrust.org). An editorial in the May issue entitled 'AGREE to disagree' recognised the important role that CPGs play in setting standards of clinical practice in SA, and introduced a formalised mechanism to assess CPG quality prior to publication. ${ }^{[8]}$

This editorial outlines and discusses key aspects of CPG quality, and sets the scene for the South African Guidelines Excellence (SAGE) project, funded for 3 years by the South African Medical Research Council. This innovative research partnership aims to improve the quality and reach of SA primary care CPGs. Using stakeholderdriven processes, SAGE will provide tools to assist effective SA CPG activities in developing, adapting, adopting, contextualising and implementing primary care CPGs.

\section{International standards for guideline developers}

Between 2011 and 2013, three standards were independently proposed, to assist CPG developers in addressing key issues of quality (Institute of Medicine (IOM) 8 standards, ${ }^{[2]}$ G-I-N 11 standards, ${ }^{[4]}$ and McMaster University group 18 standards $\left.{ }^{[9]}\right)$. Concurrently, two checklists were independently developed to appraise CPG quality. The AGREE II checklist (Appraisal of Guideline ResEarch and Evaluation) uses six domains incorporating 23 items (each scored $1-7$ ), ${ }^{[10]}$ while the iCAHE checklist (International Centre for Allied Health Evidence) provides a simpler alternative for policy makers and clinicians, with seven domains incorporating 14 binary items. ${ }^{[11]}$ Table 1 compares the items in each checklist, using the AGREE II domains to standardise comparison. Domains common to all instruments are 'stakeholder involvement,' 'underlying evidence', 'currency' and 'clarity'.

Stakeholder involvement. Stakeholder (end-user) involvement directly links CPGs to ownership, and downstream implementation. It is therefore an essential initial step to identify all relevant stakeholders within a CPG's scope and purpose, and then determine the role each stakeholder might play in the CPG development process. This assists determination of clear terms of reference. Stakeholder engagement can either occur individually ('experts' working with the methodology team) or as a collective (providing feedback on CPG drafts, or at public consultations). 


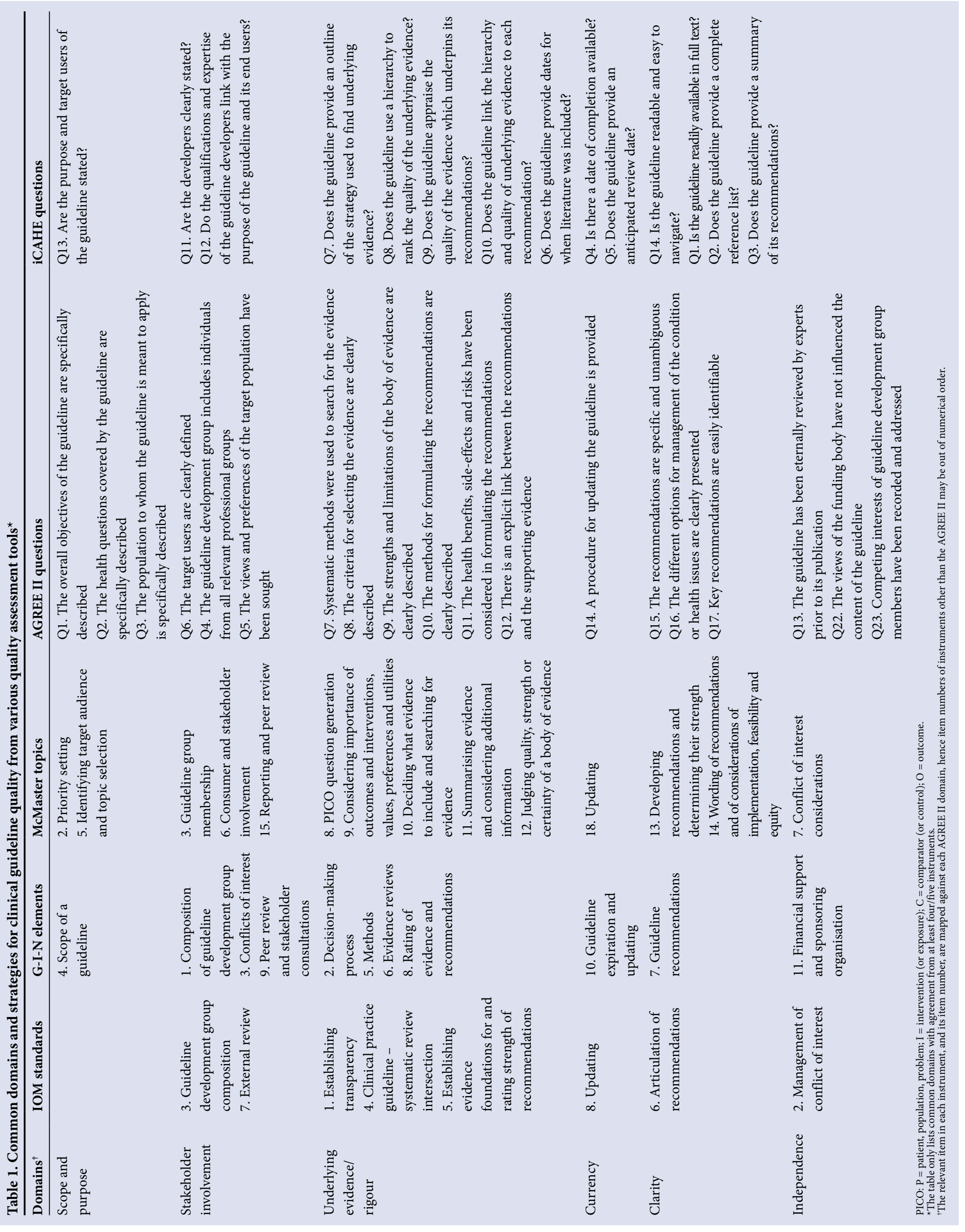


Scope and purpose. The CPG purpose intrinsically links with endusers and the target audience (people to whom the guidance is being directed). The CPG scope also underpins the framing of the research questions. For instance, for a CPG aimed at primary care clinicians, research questions would not be raised about care provided in other sectors. Defining scope and purpose early, and clearly, assists in determining which stakeholders need to be engaged, how, and in what ways.

Independence. It is critical that everyone involved in CPG development is identified, their qualifications listed and their role on the guideline team described, and potential conflicts of interest declared in writing throughout the CPG activity. Funding for the $\mathrm{CPG}$ and endorsements should be stated clearly. ${ }^{[12]}$ Independence is essential when sourcing and critiquing the evidence, so that one person's or group's view of the literature does not dominate. ${ }^{[13]}$

Underlying evidence. A good-quality CPG should include a comprehensive 'Methods' section, which outlines the research questions, how the literature was accessed (databases, search terms/ key words, inclusion/exclusion criteria), how the research was critiqued (hierarchy of evidence, critical appraisal tools), how data were extracted, and how the strength of the body of evidence was determined and reported for each recommendation. A comprehensive reference list of included papers should be provided, so that end-users can identify literature underpinning each recommendation.

Currency. With an estimated 1.8 million peer-reviewed articles published in academic journals by the end of $2012,^{[14]}$ ensuring that CPGs are based on current evidence is a constant challenge. This requires regular updating, using the protocols established during initial CPG development. Before updating, CPG developers should first identify new issues that have arisen since the previous CPG was published. They should also consider the relevance of the questions 'carried forward' from the last CPG. Literature searches should be undertaken from the date of completion of the previous search to the present, to update the evidence base. The relevance of any new findings should be factored into previous recommendations, using a standard approach. ${ }^{[1]}$

Clarity. Clearly written CPGs and comprehensive supporting documentation are essential to ensure that end-users can be confident that they can trust the recommendations. This reduces barriers to uptake and implementation. ${ }^{[2]}$ Moreover, the use of standard clear wording when writing recommendations is encouraged, to clearly link the strength of the evidence body with the wording of the recommendation. ${ }^{[6,15]}$

\section{Conclusion}

In this editorial, the first in a series of six, we present issues critical to CPG development and uptake, relevant to SA and beyond. While recent local efforts to improve CPG quality and credibility in SA are commendable, ${ }^{[8,7]}$ opportunities to progress SA CPG quality and uptake are limited by the lack of a central, nationally recognised and accepted CPG development unit. Such a unit has the potential to significantly increase SA efforts to improve and standardise high-quality, credible CPG development, reporting and uptake. To this end, the Project SAGE team is engaging in a 3-year stakeholder-driven process that aims to better understand the guideline development arena in SA, and improve the standard of local guideline development, adaptation, contextualisation, and ultimately implementation of primary healthcare guidelines.

\section{Shingai Machingaidze, Tamara Kredo \\ Cochrane South Africa, South African Medical Research Council, Cape Town, South Africa}

\section{Quinette Louw}

Department of Physiotherapy, Faculty of Medicine and Health Sciences, Stellenbosch University, Tygerberg, Cape Town, South Africa, and Cochrane South Africa, SA Medical Research Council, Cape Town

\section{Taryn Young}

Centre for Evidence-Based Health Care, Faculty of Medicine and Health Sciences, Stellenbosch University, Tygerberg, Cape Town, South Africa, and Cochrane South Africa, SA Medical Research Council, Cape Town

\section{Karen Grimmer}

International Centre for Allied Health Evidence, University of South Australia, Adelaide, Australia, and Department of Physiotherapy, Faculty of Medicine and Health Sciences, Stellenbosch University, Tygerberg, Cape Town, South Africa

Corresponding author: S Machingaidze (shingai.machingaidze@mrc.ac.za)

1. Field MJ, Lohr KN. Clinical Practice Guidelines: Directions for a New Program. Washington, DC:

National Academy Press, 1990.
2. Graham R, Mancher M, Wolman D, Greenfield S, Steinberg E. Clinical Practice Guidelines We Can

2. Graham R, Mancher M, Wolman D, Greenfield S, Steinberg E. Clinical Practice Guidelines We Can
Trust. Washington, DC: Institute of Medicine Committee on Standards for Developing Trustworthy Trust. Washington, DC: Institute of Medicine Committee on ST
Clinical Practice Guidelines/National Academies Press, 2011:15.

3. Clearing House, International Centre for Allied Health Evidence (UniSA). http://www.unisa.edu.au/ Research/Sansom-Institute-for-Health-Research/Research-at-the-Sansom/Research-Concentrations/ Allied-Health-Evidence/Resources/GuidelineCH/Low-Back-Pain-Guidelines/ (accessed 28 April 2015).

4. Guidelines International Network (G-I-N). http://www.g-i-n.net/ (accessed 28 April 2015).

5. Guyatt GH, Oxman AD, Vist GE, Falck-Ytter Y, Alsonso-Coello P, Schünemann HJ, and the GRADE working group. GRADE: An emerging consensus on rating quality of evidence and strength of recommendations. BMJ 2008;336:924. [http://dx.doi.org/10.1136/bmj.39489.470347.AD]

6. Treweek S, Oxman A, Alderson P, et al. and the DECIDE Consortium. Developing and evaluating communication strategies to support informed decisions and practice based on evaluating communication strategies to support informed decisions and practice based on
evidence (DECIDE): Protocol and preliminary results. Implement Sci 2014;8:6. [http://dx.doi. evidence (DECIDE): Protoco
org/10.1186/1748-5908-8-6]

7. Kredo T, Gerritsen A, van Heerden J, Conway S, Siegfried N. Clinical practice guidelines within Kredo T, Gerritsen A, van Heerden J, Conway S, Siegfried N. Clinical practice guidelines within
the Southern African Development Community: A descriptive study of the quality of guideline the Southern African Development Community: A descriptive study of the quality of guideline
development and concordance with best evidence for five priority diseases. Health Res Policy Syst

2012;10:1. [http://dx.doi.org/10.1186/1478-4505-10-1]
8. Wiseman R, Cohen K, Gray A, et al. AGREE to disagree: Critical appraisal and the publication of practice guidelines. S Afr Med J 2014;104(5):345-346. [http://dx.doi.org/10.7196/samj.8215]

9. McMaster Guideline Development Checklist. http://cebgrade.mcmaster.ca/guidelinechecklistonline. html (accessed 28 April 2015).

10. Brouwers M, Kho ME, Browman GP, et al. for the AGREE Next Steps Consortium. AGREE II: Advancing guideline development, reporting and evaluation in healthcare. Can Med Assoc J 2010;82(18):E839-E842. [http://dx.doi.org/10.1503/cmaj.090449]

11. Grimmer K, Dizon J, Milanese S, et al. Efficient clinical evaluation of guideline quality: Development and testing of a new tool. BMC Res Notes 2014;14:63. [http://dx.doi. org/10.1186/1471-2288-14-63]

12. Lurie P, Almeida CM, Stine N, Stine AR, Wolfe SM. Financial conflict of interest disclosure and Lurie $\mathrm{P}$, Almeida $\mathrm{CM}$, Stine $\mathrm{N}$, Stine AR, Wolfe SM. Financial conflict of interest disclosure and
voting patterns at Food and Drug Administration Drug Advisory Committee meetings. JAMA 2006;295(16):1921-1928. [http://dx.doi.org/10.1001/jama.295.16.1921]

13. Shea BJ, Grimshaw JM, Wells GA, et al. Development of AMSTAR: A measurement tool to assess the methodological quality of systematic reviews. BMC Med Res Methodol 2007;7:10. [http://dx.doi. org/10.1186/1471-2288-7-10]

14. Ware M, Mabe M. The STM Report: An Overview of Scientific and Scholarly Journal Publishing. The Hague: STM: International Association of Scientific, Technical and Medical Publishers, 2012. 15. Shiffman RN, Dixon J, Brandt C, et al. The GuideLine Implementability Appraisal (GLIA): Development of an instrument to identify obstacles to guideline implementation. BMC Med Inform Decis Mak 2005;5:23. [http://dx.doi.org/10.1186/1472-6947-5-23]

S Afr Med J 2015;105(9):743-745. DOI:10.7196/SAMJnew.7697 